\title{
Effect of Administering Freund's Complete Adjuvant to Spinal Nerves on the Development of Allodynia in the Rat
}

\author{
Department of Anesthesiology and Pain Medicine, Asan Medical Center, University of Ulsan College of Medicine, Seoul, Korea
}

Jong Yeon Park, M.D., and Jai Hyun Hwang, M.D.

Background: Spinal nerve ligation injury causes a neuropathic pain syndrome that includes allodynia. Neuropathic pain is also induced by Freund's complete adjuvant (FCA)-induced inflammation. This study was designed to examine the development of mechanical and cold allodynia after FCA administration at the L4/L5 spinal nerves and to compare it with the effects of spinal nerve ligation at the same site.

Methods: Rats were randomly allocated into three groups: (i) treatment with tight ligation of the left L5/L6 spinal nerves, (ii) wrapping of the L5/L6 spinal nerves with Spongostan ${ }^{R}$ soaked in FCA, or (iii) wrapping of the L5/L6 spinal nerves with Spongostan ${ }^{\mathbb{R}}$ soaked in saline. Mechanical and cold allodynia were measured by applying von Frey filaments or acetone in both hind paws. To examine the development of allodynia, the frequencies of hind paw withdrawal to each type of stimulus were measured.

Results: Both FCA administration and nerve ligation injury caused a marked mechanical and cold allodynia in the lesioned hind paw compared to saline treatment $(\mathrm{P}<0.05)$. Furthermore, the frequencies of response of the lesioned hind paw to both types of stimulus were significantly greater than those on the contralateral side.

Conclusions: These results suggest that administration of FCA to the spinal nerves can produce a mechanical and cold allodynia with a similar profile of pain facilitation as nerve ligation. (Korean J Anesthesiol 2007; 53: S 36 41)

Key Words: allodynia, cold, mechanical, FCA, rat, spinal nerve ligation.

\section{INTRODUCTION}

Peripheral nerve injury can cause extreme cutaneous sensitivity to normally innocuous mechanical and cold stimuli, a condition called allodynia. Unilateral ligation of lumbar L5/L6 spinal nerves produces neuropathic pain, and may produce a profound allodynia that lasts for several weeks. ${ }^{1-3)}$ Amongst various experimental animal models, signs of tactile allodynia are most evident in the nerve ligation model. ${ }^{2)}$ Freund's complete adjuvant (FCA), an inflammatory agent, is widely used in animal models of chronic pain. ${ }^{4-6)}$ Although FCA-induced inflammation produces a neuropathic pain, many investigators have also used

Received : April 27, 2007

Corresponding to : Jai Hyun Hwang, Department of Anesthesiology and Pain Medicine, Asan Medical Center, 388-1, Pungnap 2-dong, Songpa-gu, Seoul 138-736, Korea. Tel: 82-2-3010-3859, Fax: 82-2-470-1363, E-mail: jhhwang@amc.seoul.kr

This study was supported by a grant (\# 03-153) from Asan Institute for Life Sciences, Seoul, Korea.
FCA to induce a peripheral inflammation. ${ }^{4-6)}$ Recent studies have shown that focal inflammation of the sciatic nerve produces neuropathic pain in distant regions by a mechanism other than axonal damage. ${ }^{7,8)}$ Thus, the researchers hypothesized that administration of FCA around the L5 and L6 spinal nerves in place of nerve ligation would generate mechanical and cold allodynia, two clinical presentations of neuropathic pain. The current study was designed to investigate this possibility and to compare the development of allodynia with that caused by nerve injury.

\section{MATERIALS AND METHODS}

All the experiments performed, according to protocols approved by our Animal Care Committee. Forty male Sprague-Dawley rats weighing $180-230 \mathrm{~g}$ were used for the experiments. The rats were housed three to four per a cage, given food and water added libitum, and raised in a room maintained temperature $21 \pm 1^{\circ} \mathrm{C}$ in a $12 / 12$-hrs light/dark. The rats were acclimatized for 3 days before surgery. Surgeries were perfor- 
med under halothane anesthesia and ventilation with $1: 1 \quad \mathrm{~N}_{2} \mathrm{O} / \mathrm{O}_{2}$. The rats recovered sufficiently from the surgical procedures to resume normal activity within $30 \mathrm{~min}$ after termination of the anesthesia. The neuropathic rat model was generated using the surgical procedure described by Kim and Chung. ${ }^{1)}$ Under anesthesia, a dorsal midline incision was made from the L3 to the S2 vertebral level. The left L6/S1 posterior interarticular process was exposed and resected. In the spinal nerve ligation (SNL) group ( $\mathrm{n}=14$ ), a partial excision of the L6 transverse process was made and the left L5 and L6 spinal nerves were gently isolated and ligated tightly with 6-0 black silk just distal to the dorsal root ganglion and proximal to the formation of the sciatic nerve. In the FCA group $(n=14)$, rats were anesthetized and the left L5 and L6 spinal nerves were isolated but not ligated. The L5 and L6 spinal nerves were then loosely wrapped with a band of Spongostan ${ }^{\circledR}(2 \times 15$ mm; Johnson \& Johnson, UK) soaked with 70 (1 of FCA (Sigma, St. Louis, MO, USA). For the saline (control) group $(n=12)$, the same procedure was performed using Spongostan ${ }^{\circledR}$ soaked in saline. After each surgical procedure, complete hemostasis was confirmed and the wound was closed by tight suturing.

Behavioral testing was performed during the day portion of the circardian rhythm (9:00 AM to 3:00 PM). Measurements were taken before and ever other days until the $21^{\text {st }}$ days after surgical procedures. To measure the frequencies of the withdrawal response, each rat was placed under a transparent plastic box on a metal mesh floor. The rats were allowed to acclimate to the environment for $10 \mathrm{~min}$ before starting the test. Four to six animals were tested simultaneously. Three types of behavioral tests representing mechanical and cold allodynia were performed. Baseline values were calculated by averaging three measurements made 1 day before each operation. To quantify mechanical sensitivity, innocuous mechanical stimuli were applied with von Frey filaments (Stoelting Co., Wood Dale, IL, USA) at two different bending forces (2.0 and 15.1 g) to the mid plantar surface of the hind paw until a positive sign of pain behavior was elicited. Each filament was applied 10 times (once every $10 \mathrm{~s}$ ) to both hind paws with sufficient force to cause slight bending against the paw and it was held for $6 \mathrm{~s}$. A brisk withdrawal, paw flinching, and/or licking of the paw were considered as positive responses. The frequency of foot withdrawals was again expressed as a percentage ([number of trials accompanied by brisk foot withdrawal $\div$ total number of trials] $\times 100$ ). Stimulation of normal human skin with the weak $(2.0 \mathrm{~g})$ and strong (15.1 g) filaments elicits a faint sense of touch and sense of pressure, respectively. Therefore, a significant increase in the frequency of brisk foot withdrawals in response to these innocuous mechanical stimuli was interpreted as mechanical allodynia. This interpretation was supported by the fact that the withdrawals frequently accompanied aversive behaviors such as shaking and licking the lifted paw. ${ }^{3,9)}$ To quantify cold sensitivity of the foot, brisk foot withdrawal in response to application of acetone was measured as described previously. ${ }^{9)}$ Application of acetone has been widely used to detect the presence of cold allodynia. ${ }^{2,9)}$ The rat was placed under a transparent plastic box on a metal mesh floor. Acetone was applied to the plantar surface of the rat's heel as a bubble formed at the end of a small piece of polyethylene tubing connected to a syringe. Once touched to the foot, the acetone quickly spread over the proximal half of the plantar surface of the foot. Acetone was applied in this way 10 times (once every $5 \mathrm{~min}$ ) to each paw. The frequency of foot withdrawal was again expressed as a percentage ([number of trials accompanied by brisk foot withdrawal $\div$ total number of trials] $\times 100$ ). A significant increase in the frequency of foot withdrawals in response to acetone application was interpreted as cold allodynia.

On each test day, the behavioral tests were carried out in the following order: weak $(2.0 \mathrm{~g})$ filament, acetone and strong $(15.1 \mathrm{~g})$ filament. The order of tests was maintained throughout the test period for all animals. Between the different tests, the animals were allowed to rest for at least $30 \mathrm{~min}$. During entire experimental period, general activity such as ambulation, weight bearing and posture was observed at every time point to check for allodynia and to identify the factors that may affect pain assessment. Simultaneously, righting reflex and placing/stepping reflexes were measured to evaluate motor weakness. Because animals in the SNL group showed a characteristic mild foot deformity of the lesioned hind paw, data could not be collected in a blinded fashion.

Data are expressed as the means \pm standard errors (SEM) in each group. Differences in changes of mean values over time were tested using repeated measures analysis of variance (ANOVA) or the Friedman ANOVA by ranks followed by post-hoc pair-wise comparisons (SigmaStat 3.1). Differences among the three groups were analyzed using one-way ANOVA or Kruskal-Wallis ANOVA by ranks followed by post-hoc comparisons for group differences. Differences between left and right withdrawal responses were analyzed using a paired t-test 
or a Mann-Whitney Rank Sum test. P values less than 0.05 were considered to indicate statistical significance.

\section{RESULTS}

After spinal nerve ligation or FCA administration, all rats displayed normal general behavior and weight gain. The results of both SNL and FCA groups showed similar courses for the development the different responses (Fig. 1-3). The maximal effects were observed within 3-7 days and then gradually returned to the baseline for each type of stimulus in both groups. In both the SNL and FCA groups, the frequency of responses of the lesioned hind paw to both evoked mechanical and cold stimuli was significantly higher than the preoperative frequency $(\mathrm{P}<0.001)$ (Fig. $1-3)$. In the saline (control) group, a stronger mechanical stimulus (Fig. 2) but not a weaker mechanical or a cold stimulus caused a significant increase in the frequency of responses over the baseline frequencies (Fig. 1, 3). Compared with the saline group, prominent mechanical and cold allodynic effects for the lesioned paw were observed for both the SNL and FCA groups $(\mathrm{P}<0.05)$. A strong tactile

\section{VFH $2.0 \mathrm{~g}$ force}

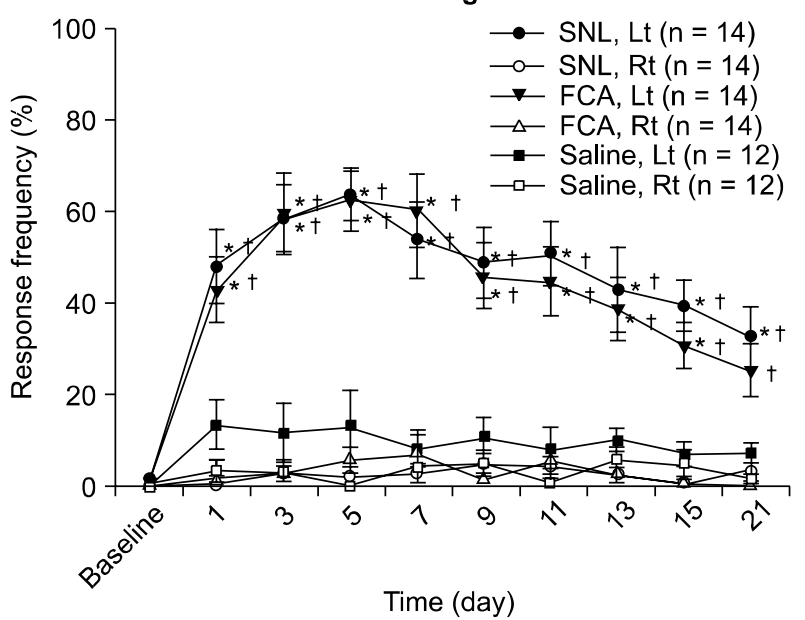

Fig. 1. Responses to repeated mechanical stimuli in each grouop. The frequencies of foot withdrawal to repeated mechanical stimuli applied to the both hind paw with von Frey filament $(2.0 \mathrm{~g})$ are expressed as percentage (mean \pm SEM) of trials. Time is expressed as baseline or postoperative day. Baseline values were calculated by averaging 3 measurements made at 1 day before each operation. $\mathrm{FCA}=$ Freund's complete adjuvant, $\mathrm{SNL}=$ spinal nerve ligation. ${ }^{*} \mathrm{P}<0.001$ indicates significant differences from the baseline value during the postoperative test period. ${ }^{\dagger} \mathrm{P}<0.05$ indicates significant differences from the saline group in the lesioned hind paw.

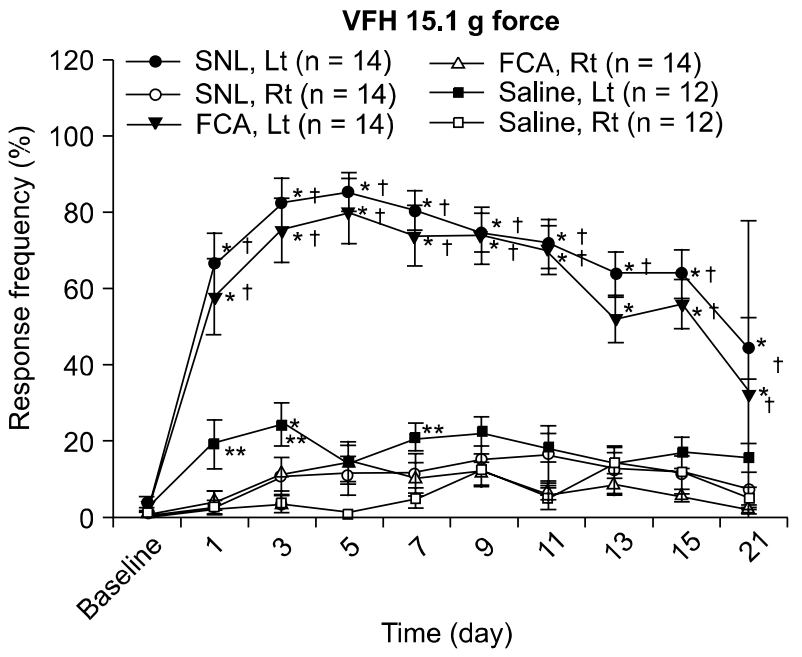

Fig. 2. Responses to repeated mechanical stimuli in each grouop. The frequencies of foot withdrawal to repeated mechanical stimuli applied to the both hind paw with von Frey filament $(15.1 \mathrm{~g})$ are expressed as percentage (mean \pm SEM) of trials. Time is expressed as baseline or postoperative day. FCA = Freund's complete adjuvant, $\mathrm{SNL}=$ spinal nerve ligation. ${ }^{*} \mathrm{P}<0.001$ indicates significant differences from the baseline value during the postoperative test period. ${ }^{*} \mathrm{P}<0.05$ indicates significant differences from contralateral right side. ${ }^{\dagger} \mathrm{P}<0.05$ indicates significant differences from the saline group in the lesioned hind paw.

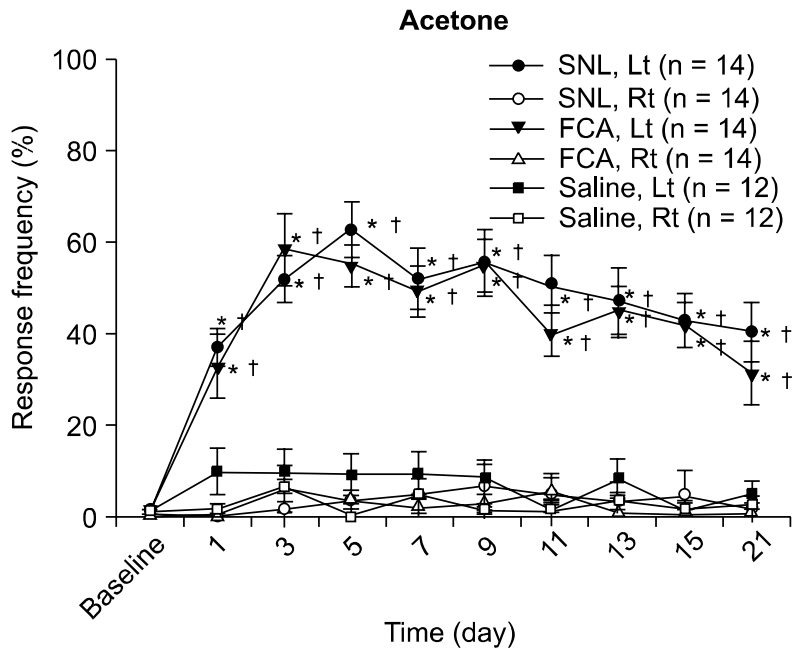

Fig. 3. Responses to repeated cold stimuli in each grouop. The frequencies of foot withdrawal to repeated cold stimuli (acetone) applied to the both hind paw are expressed as percentage (mean \pm SEM) of trials. Time is expressed as baseline or postoperative day. $\mathrm{FCA}=$ Freund's complete adjuvant, $\mathrm{SNL}=$ spinal nerve ligation. ${ }^{*} \mathrm{P}<0.001$ indicates significant differences from the baseline value during the postoperative test period. ${ }^{\dagger} \mathrm{P}<0.05$ indicates significant differences from the saline group in the lesioned hind paw. 
stimulus $(15.1 \mathrm{~g})$ caused a greater increase in the frequency of responses in both groups than a weak stimulus (Fig. 2). Likewise, both groups showed a statistically significant difference in the withdrawal response to a cold stimulus on the ipsilateral lesioned hind paw $(\mathrm{P}<0.001)$ (Fig. 3). The response frequencies of the lesioned hind paw to each type of stimulus were significantly higher than those of the contralateral side in all groups, including the saline group $(\mathrm{P}<0.05)$. None of the groups showed an allodynic response to any of the stimuli on the contralateral right hind paw when compared with the preoperative responses.

The level of general activities was indistinguishable from that of normal rats. Abnormal behavior or foot deformities were not seen in the saline or FCA groups, whereas most rats in the SNL group showed a slight foot deformity of the lesioned hind paw after surgery. The foot of the lesioned side in these rats was moderately everted on the injured side and the toes were held together. All of the animals in both the SNL and FCA groups had allodynic responses without effects on general activity or any signs of motor weakness.

\section{DISCUSSION}

In this study, we made two important observations: First, administration of FCA around the L5 and L6 spinal nerves can produce both mechanical and cold allodynia as a result of nerve injury. Second, inflammation of the spinal nerves created pain facilitation as by nerve ligation. In brief, both manipulations increased the frequency of withdrawal responses by either a tactile or cold stimulus, suggesting that treatment of spinal nerves with FCA causes allodynia. Kim and Chung ${ }^{1)}$ reported that mechanical allodynia in the affected foot as a result of spinal nerve ligation lasts for several weeks. In the present study, the allodynic response to tactile stimuli on the unilateral affected side was maintained for one or up to 3 weeks.

Experimental animal models of nerve injury have been mostly generated by simple spinal nerve ligation, partial sciatic nerve ligation or chronic sciatic nerve constriction., ${ }^{1,10,11)}$ Unilateral ligation of lumbar L5 and L6 spinal nerves produces some signs that appear to be representative of neuropathic pain $^{1-3,12)}$ as well as a profound allodynia that lasts for several weeks. ${ }^{1)}$ A previous study showed that of several experimental animal models, tactile allodynia was most evident in the nerve ligation model. ${ }^{2)}$
FCA is widely used to generate models of chronic inflammatory pain. ${ }^{4-6,13)}$ The precise mechanism of neuropathic pain caused by FCA in the current study is not clear, but it is possible that inflammatory mediators sensitize acutely inflamed nerve fibers to mechanical and thermal stimuli. ${ }^{14,15)}$ Another probable mechanism is that an inflammation-induced neuropathy (neuritis) caused by FCA initiates an immune response and produces a local destructive caseous local inflammation with marked granuloma. ${ }^{7)}$ Therefore, nonspecific mechanisms, such as nerve compression by the surrounding granuloma or direct destruction of the nerve by the inflamed caseous tissue, may participate in the development of the allodynia. ${ }^{7}$

Several studies have reported that a neuroimmune interaction contributes to the genesis of painful peripheral neuropathies. ${ }^{7,8,16}$ An inflammation reaction, immune cell infiltration and increased endoneurial levels of pro-inflammatory cytokines have been detected at the site of nerve injury in animal models of painful peripheral neuropathy. ${ }^{17-19)}$ Watkins et al. ${ }^{20)}$ demonstrated that the neuropathic pain produced by the neuritis is accompanied by minor structural damage to axons or glia and that application of FCA to the surface of the nerve evokes an endoneurial inflammation characterized by evidence for plasma extravasation and the infiltration of immune cells. ${ }^{20)}$ Also, Maves et al. ${ }^{21)}$ suggested that a neuroimmune interaction in the endoneurial compartment plays a key role in producing the neuritis-evoked neuropathic pain. Therefore, we believe that inflammation subsequent to either FCA administration or surgical tissue injury is sufficient to induce the development of allodynia.

A recently developed model of sciatic inflammatory neuritis was shown to induce unilateral and bilateral mechanical allodynia following acute unilateral peri-sciatic immune activation in rats, ${ }^{8)}$ whereas a focal inflammation of the sciatic nerve was shown to produce neuropathic pain on the ipsilateral side. ${ }^{7)}$ In constant to the studies by Eliav et al. ${ }^{7)}$ in our experiments, FCA induced a significant unilateral mechanical and cold allodynia. However, the duration of persistent pain induced by FCA was lasted up to 3 weeks.

Although the site of FCA administration was different than in previous animal studies, ${ }^{4,22)}$ and signs of external inflammation were not observed, we observed behavioral signs of neuropathic pain within one day after operation. Following intraplantar injection of FCA, Stein et al. ${ }^{22)}$ observed apparent inflammation of the affected paw within $12 \mathrm{~h}$, whereas Leem et al. ${ }^{4}$ observed characteristic inflammatory signs 2 days after 
the injection of FCA. Field et al. $^{23)}$ demonstrated that the static component of mechanical allodynia, which is assessed by application of von Frey hair, developed more rapidly in FCA-injected animals than in those with constriction nerve injury and that it could be observed on the first operative day. Our findings in SNL rats are consistent with these previous reports. Also, mechanical allodynia was observed on the first day after treatment in the FCA group, as found in the previous studies.

Because the contralateral side could also be affected by the treatments, we checked the response to each stimulus by both hind paws. Several previous studies have observed that following peripheral nerve lesions, there are events that affect the contralateral non-lesioned structures. ${ }^{24,25)}$ In this study, however, the treatments had little effect on the frequency of responses to each type of stimulus on the contralateral side. There was a slight increase in the response frequency for the left hind paw in the saline group that might reflect experimental bias.

Whether FCA was confined to the L5/L6 spinal nerves and whether it affected the L4 spinal nerve following administration of the FCA- saturated gelfoam is not clear. An effect on the L4 spinal nerve might impair motor activity, but we did not observe a change in motor activity, possibly because the concentration of FCA at the L4 spinal nerve was too low. Regardless, L4 damage does not have a relevant role at the level of the responses measured in the current study, but it may be worth considering its contribution in future experiments.

When using either chronic constriction nerve injury or partial nerve ligation as a model of neuropathic pain rat model, ${ }^{10,11)}$ marked deformity of the lesioned hind paw causes abnormal weight bearing which can bias a blinded experimenter. Abnormal weight bearing of the limb is a confounding factor for the assessment of mechanical allodynia in behavioral studies. Kauppila et al. ${ }^{26)}$ demonstrated that withdrawal thresholds were significantly lower when the hind limbs were not bearing weight. In rats with a unilateral nerve ligation, the decrease in the threshold on the side ipsilateral to the nerve lesion was independent of the weight bearing by the hind limbs. ${ }^{26)}$

The spinal L5 and L6 nerve ligation model mostly produced mechanical allodynia. It is possible, however, to determine which is the lesioned hind paw in this model. This can bias the experimenter's ability to measure the responses. Although the foot deformity in the spinal ligation model is minor, the problem of abnormal weight bearing to be resolved. In contrast, none of the rats in the FCA group showed foot deformities on the lesioned side. This is an advantage over the SNL model because it avoids the problem of experimental bias. Thus, we believe that the FCA model of allodynia can avoid the problem of foot deformity in the SNL model.

On the other hand, we did not consider the surgical procedure itself as a factor that can affect the development and maintenance of allodynia because the results of responses to weak mechanical and cold stimuli did not have substantial effects in the saline group. Unexpectedly, however, a strong mechanical stimulus increased the frequency of responses in this group. Although a strong filament $(15.1 \mathrm{~g})$ elicits a sense of pressure in the human skin, this is not considered a nociceptive sensation. Also, it does not appear to have a hyperalgesic effect because it is not a noxious stimulant as shown in the preoperative test. It is concluded that the responses to the weaker stimuli were more meaningful.

In general, acetone or a cold plate is used to test for cold allodynia. $^{2,9-11)}$ However, it did not completely eliminate the component of touch. To reduce the influence of touch, we applied an acetone bubble by gently touching it to the heel, after which the acetone quickly spread over the proximal half of the plantar surface of the foot.

Despite precautions, there were some limitations that we considered when interpreting the results. First, we did not assess the histology, assays for potential mediators, or perform pharmacologic or other interventions because the aim of this study was to determine whether FCA administration could initiate and maintain allodynia. Also, we did not examine the correlation between behavioral signs of allodynia and inflammatory changes in the spinal nerve although we found that inflammation of the spinal nerves caused a similar profile of pain facilitation as nerve ligation.

Present study results support the hypothesis that FCA administration around the spinal nerves produces neuropathy. However, this model should be verified by histological studies because there are different phenotypic changes that occur following inflammation and nerve injury. Second, we did not examine the ongoing spontaneous pain, which is a typical sign of neuropathic pain, and we checked the static component of mechanical allodynia only. Third, we used two von Frey filaments for the mechanical stimulus. Furthermore, pharmacological studies with both positive and negative compounds are needed to confirm that FCA administration causes neuropathy rather than other conditions because mechanical and cold allodynia can also be symptoms of other conditions besides 
neuropathy. Pharmacological validation of this new technique is needed to draw clearer conclusions using this model.

In conclusion, we showed that local administration of FCA on the L5 and L6 spinal nerves induces mechanical and cold allodynia without any external deformity. The mechanical and cold allodynic states generated in this model are equivalent to those caused by spinal nerve ligation injury. Therefore, it appears that FCA administration around the spinal nerves can serve as an alternative model of neuropathic pain.

\section{REFERENCES}

1. Kim SH, Chung JM: An experimental model for peripheral neuropathy produced by segmental spinal nerve ligation in the rat. Pain 1992; 50: 355-63.

2. Kim KJ, Yoon YW, Chung JM: Comparison of three rodent neuropathic pain models. Exp Brain Res 1997; 113: 200-6.

3. Sheen K, Chung JM: Signs of neuropathic pain depend on signals from injured nerve fibers in a rat model. Brain Res 1993; 610: $62-8$.

4. Leem JW, Hwang JH, Hwang SJ, Park H, Kim MK, Choi Y: The role of peripheral N-methyl-D-aspartate receptors in Freund's complete adjuvant induced mechanical hyperalgesia in rats. Neurosci Lett 2001; 297: 155-8.

5. Millan MJ, Czlonkowski A, Morris B, Stein C, Arendt R, Huber $\mathrm{A}$, et al: Inflammation of the hind limb as a model of unilateral, localized pain: influence on multiple opioid systems in the spinal cord of the rat. Pain 1988; 35: 299-312.

6. Stein C, Millan MJ, Herz A: Unilateral inflammation of the hindpaw in rats as a model of prolonged noxious stimulation: alterations in behavior and nociceptive thresholds. Pharmacol Biochem Behav 1988; 31: 455-51.

7. Eliav E, Herzberg U, Ruda MA, Bennett GJ: Neuropathic pain from an experimental neuritis of the rat sciatic nerve. Pain 1999; 83: $169-82$

8. Chacur M, Milligan ED, Gazda LS, Armstrong C, Wang H, Tracey $\mathrm{KJ}$, et al: A new model of sciatic inflammatory neuritis (SIN): induction of unilateral and bilateral mechanical allodynia following acute unilateral peri-sciatic immune activation in rats. Pain 2001; 94: $231-44$

9. Choi Y, Yoon YW, Na HS, Kim SH, Chung JM: Behavioral signs of ongoing pain and cold allodynia in a rat model of neuropathic pain. Pain 1994; 59: 369-76.

10. Seltzer Z, Dubner R, Shir Y: A novel behavioral model of neuropathic pain disorders produced in rats by partial sciatic nerve injury. Pain 1990; 43: 205-18.

11. Bennett GJ, Xie YK: A peripheral mononeuropathy in rat that produces disorders of pain sensation like those seen in man. Pain
1988; 33: 87-107.

12. Carlton SM, Lekan HA, Kim SH, Chung JM: Behavioral manifestations of an experimental model for peripheral neuropathy produced by spinal nerve ligation in the primate. Pain 1994; 56: $155-66$.

13. LaBuda CJ, Fuchs PN: A behavioral test paradigm to measure the aversive quality of inflammatory and neuropathic pain in rats. Exp Neurol 2000; 163: 490-4.

14. Michaelis M, Vogel C, Blenk KH, Arnarson A, Janig W: Inflammatory mediators sensitize acutely axotomized nerve fibers to mechanical stimulation in the rat. J Neurosci 1998; 18: 7581-7.

15. Sweitzer SM, Colburn RW, Rutkowski M, DeLeo JA: Acute peripheral inflammation induces moderate glial activation and spinal IL-1beta expression that correlates with pain behavior in the rat. Brain Res 1999; 829: 209-21.

16. Bennett GJ: Does a neuroimmune interaction contribute to the genesis of painful peripheral neuropathies? Proc Natl Acad Sci USA 1999; 96: 7737-8.

17. DeLeo JA, Colburn RW, Rickman AJ: Cytokine and growth factor immunohistochemical spinal profiles in two animal models of mononeuropathy. Brain Res 1997; 759: 50-7.

18. Clatworthy AL, Illich PA, Castro GA, Walters ET: Role of peri-axonal inflammation in the development of thermal hyperalgesia and guarding behavior in a rat model of neuropathic pain. Neurosci Lett 1995; 184: 5-8.

19. Sommer C, Myers RR: Vascular pathology in CCI neuropathy: a quantitative temporal study. Exp Neurol 1996; 141: 113-9.

20. Watkins LR, Maier SF, Goehler LE: Immune activation: the role of pro-inflammatory cytokines in inflammation, illness responses and pathological pain states. Pain 1995; 63: 289-302.

21. Maves TJ, Pechman PS, Gebhart GF, Meller ST: Possible chemical contribution from chromic gut sutures produces disorders of pain sensation like those seen in man. Pain 1993; 54: 57-69.

22. Stein C, Millan MJ, Shippenberg TS, Peter K, Herz A: Peripheral opioid receptors mediating antinociception in inflammation. Evidence for involvement of mu, delta and kappa receptors. J Pharmacol Exp Ther 1989; 248: 1269-75.

23. Field MJ, Bramwell S, Hughes J, Singh L: Detection of static and dynamic components of mechanical allodynia in rat models of neuropathic pain: are they signalled by distinct primary sensory neurones? Pain 1999; 83: 303-11.

24. Koltzenburg M, Wall PD, McMahon SB: Does the right side know what the left is doing? Trends Neurosci 1999; 22: 122-7.

25. Oaklander AL, Romans K, Horasek S, Stocks A, Hauer P, Meyer RA: Unilateral postherpetic neuralgia is associated with bilateral sensory neuron damage. Ann Neurol 1998; 44: 789-95.

26. Kauppila T, Kontinen VK, Pertovaara A: Weight bearing of the limb as a confounding factor in assessment of mechanical allodynia in the rat. Pain 1998; 74: 55-9. 